\title{
$B$ Physics Prospects at Hadron Colliders
}

\section{Barry Wicklund*}

Argonne National Laboratory, Argonne, IL, USA 60439

E-mail: abaḅanl.govi

AbSTRACT: This talk is a brief summary of the $B$ physics prospects anticipated over the next decade for experiments at the Fermilab Tevatron and the CERN Large Hadron Collider. Of particular interest is the complementarity of these experiments with those at the $\Upsilon(4 S) B$-factories

\section{Introduction}

A rich program of $B$ physics is expected over the next decade at the Fermilab and CERN hadron colliders. This program will exploit the very large $B$-production cross sections in $\bar{p} p$ and $p p$ collisions; it will also provide a unique window on the $B_{s}$ meson sector, thus providing an essential complement to the $\Upsilon(4 S) B$-factories. The Tevatron experiments, CDF and D0, are currently in commission, and will accumulate roughly $15 \mathrm{fb}^{-1}$ before LHC turnon. Later in the decade, two dedicated $B$-hadron experiments, BTeV at the Tevatron and $\mathrm{LHCb}$ at $\mathrm{LHC}$, are planned with superior triggering, data acquisition, and particle identification. In addition, the general purpose LHC experiments, ATLAS and CMS, will focus on $B$ physics measurements during the lower luminosity LHC startup. The physics goals, as for the $B$ factories, are to overconstrain the standard model CKM matrix by precision measurements of the $C P$-violation angles, $2 \beta, 2 \beta_{s}, \gamma$; the $B_{s}$ mixing amplitudes, $M_{12}$ and $\Gamma_{12}$, and rare decay rates and asymmetries. Extensive theoretical and experimental studies on all of these topics can be found in the CERN LHC [i] and Fermilab Tevatron [2] 2 ] workshop "Yellow Books", which are the primary sources for this talk.

\section{Experimental Issues}

The primary experimental issue at hadron colliders is efficent triggering. Whereas the $B \bar{B}$ production rate is $10 \mathrm{~Hz}$ at the $B$-factories at $\mathcal{L}=10^{34}$, it is approximately $20 \mathrm{kHz}$ at

\footnotetext{
${ }^{*}$ Speaker.
} 
the Tevatron and $100 \mathrm{kHz}$ at LHC at $\mathcal{L}=210^{32}$; this is accompanied by QCD backgrounds that are $\sim 1000 \times$ larger. Thus, even with kinematic restrictions $|y|<1, p_{T}(B)>6 \mathrm{GeV} / \mathrm{c}$, the $B$ rate would be $2.5 \mathrm{kHz}$ at $\mathrm{CDF}$, substantially more than the peak rate to tape of $75 \mathrm{~Hz}$. One approach common to all hadron experiments is the use of efficient single and dilepton triggers, for example for $J / \psi \rightarrow \ell^{+} \ell^{-}$and rare decay modes; this gives a big statistical advantage over the $B$ factories for these channels because of the low $B R$. A second approach adopted by $\mathrm{CDF}, \mathrm{BTeV}$, and $\mathrm{LHCb}$ is the use of high precision vertex triggers. This allows typically $\times 100$ enrichment in the $B \bar{B}$ fraction at the trigger level, and is efficient for hadronic decay modes such as $B^{0} \rightarrow \pi^{+} \pi^{-}$or $B_{s}^{0} \rightarrow D_{s}^{-} \pi^{+}$. For example, the CDF trigger, which has been demonstrated in recent commissioning, would yield a total rate of $10^{8} B \bar{B}$ events per $\mathrm{fb}^{-1}$ with roughly $1: 1$ signal:background, comparable to the $B \bar{B}$ production rate per year at $\mathcal{L}=10^{34}$ at the $B$ factories. We note that while the general purpose detectors have limited data output bandwidth of typically $\leq 100 \mathrm{~Hz}$ for all physics triggers, the $\mathrm{BTeV}$ and $\mathrm{LHCb}$ experiments are aiming for 1000 and $200 \mathrm{~Hz}$ to tape, respectively, dedicated solely to heavy flavor physics.

Particle identification is critical for channels such as $B^{0} \rightarrow \pi^{+} \pi^{-}, K^{+} \pi^{-}$and $B_{s}^{0} \rightarrow$ $D_{s}^{-} K^{+}$. CDF has time of flight and relativistic rise $d E / d x$ which provides low $p_{T}$ kaon separation and statistical separation for $B^{0} \rightarrow \pi^{+} \pi^{-} / K^{+} \pi^{-}$. BTeV and $\mathrm{LHCb}$ will have excellent $\pi-K$ separation over the full momentum range needed for the forward geometry, essential for channels like $B_{s}^{0} \rightarrow D_{s}^{-} K^{+}$. These detectors also feature excellent electromagnetic calorimetry for identification of channels such as $B_{s}^{0} \rightarrow J / \psi \eta$ and $B^{0} \rightarrow \pi^{+} \pi^{-} \pi^{0}$.

\section{Measurement of $C P$ asymmetries: $\sin 2 \beta$ and $\gamma$}

The major theme in $B$ physics is testing the consistency of the sides and angles of the unitarity triangle(s) with precision measurements. A secondary theme is direct search for non-standard model physics, such as large $C P$ asymmetries in $B_{s}^{0}$ mixing or anomalous rare decay rates. The crucial precision measurements are: the angles $\beta$ and $\gamma$, from $C P$ asymmetries, and the sides $\frac{\left|V_{u b}\right|}{\left|V_{c b}\right|}$ and $\frac{\left|V_{t d}\right|}{\left|V_{t s}\right|}$ from $b \rightarrow u$ transitions and $B^{0}$ and $B_{s}^{0}$ mixing. Of these, $\sin 2 \beta$ and $\frac{\left|V_{t d}\right|}{\left|V_{t s}\right|}$ can be measured with high experimental precision and minimal theoretical uncertainty. As is well known, the other two parameters, $\frac{\left|V_{u b}\right|}{\left|V_{c b}\right|}$ and $\gamma$ are difficult both experimentally and theoretically.

Precision measurement of $\sin 2 \beta$, using the time-dependent $C P$ asymmetry in $B^{0} \rightarrow$ $J / \psi K_{S}^{0}$, requires high statistics, good flavor tagging efficiency, and precise calibration of the flavor tagging dilution $D(D=1-2 \mathcal{W}$, where $\mathcal{W}=$ mistag rate). The hadron collider experiments can compete favorably with the $B$ factories in signal yield, but have intrinsically poorer tagging efficiency ( $\epsilon D^{2}$ "). Table $\underline{I}_{1}^{1}$ summarizes the $\sin 2 \beta$ reach projected for the hadron collider experiments $\left[\begin{array}{l}1 \\ 1\end{array}\right.$, ind , together with current $\mathrm{BaBaR}$ results.

Thus, it should be possible to measure a world-average $\sin 2 \beta$ to \pm 0.01 accuracy. At this level of precision, it will be important to resolve the four-fold ambiguity on $\beta$ (for example, via interference asymmetries in $\left.J / \psi K^{* 0}\right)$ and to verify that the direct asymmetry, proportional to $\cos \Delta M t$, is consistent with the standard model expectation. Besides test- 


\begin{tabular}{|l|r|r|r|l|}
\hline Exp. & $\sigma(\sin 2 \beta)$ & $N_{\psi K_{s}}$ & $\epsilon D^{2}$ & Luminosity \\
\hline CDF & 0.045 & $28 \mathrm{~K}$ & 0.091 & $2 \mathrm{fb}^{-1}$ \\
DO & .040 & $34 \mathrm{~K}$ & 0.10 & $2 \mathrm{fb}^{-1}$ \\
BTeV & 0.025 & $80 \mathrm{~K}$ & 0.10 & 1 Yr. $\times 210^{32}$ \\
LHCb & 0.021 & $88 \mathrm{~K}$ & 0.064 & 1 Yr. $\times 210^{32}$ \\
CMS & 0.015 & $433 \mathrm{~K}$ & 0.054 & 1 Yr. $\times 10^{33}$ \\
ATLAS & 0.017 & $165 \mathrm{~K}$ & 0.033 & 1 Yr. $\times 10^{33}$ \\
BaBaR & 0.149 & $(1030)$ & 0.261 & $3210^{6} B \bar{B}$ \\
\hline
\end{tabular}

Table 1: Projected $\sin 2 \beta$ reach. Only a subset of tags are used in ATLAS and LHCb studies.

ing the consistency of the unitarity triangle, the phase $2 \beta$ is a critical input for measurement of $\gamma$ in $B^{0} \rightarrow \pi^{+} \pi^{-}, \pi^{+} \pi^{-} \pi^{0}$.

Experimental determination of the angle $\gamma\left(=\arg V_{u b}\right.$ in the Wolfenstein representation) is more difficult, and theoretical uncertainties are such that it will be necessary to demonstrate consistent results in a variety of channels. Three prominent channels have been explored, for which hadron colliders have unique capabilities.

(1) $\gamma$ from $B_{s}^{0} \rightarrow D_{s}^{ \pm} K^{\mp}$ : this channel is unique to hadron colliders. It almost certainly will require the quality of $K / \pi$ separation planned for $\mathrm{BTeV}$ and $\mathrm{LHCb}$. It has the advantage that the decays are expected to be predominantly tree-level and impervious to new physics from penguin diagrams. It relies on the fact that $B_{s}^{0}$ and $\overline{B_{s}^{0}}$ can each decay to both $D_{s}^{ \pm} K^{\mp}$ final states, giving four time-dependent decay distributions. Because the final states are flavor specific, the time dependence includes ordinary mixing as well as $C P$ asymmetry. For example, using current estimates of the Wolfenstein parameters, the expected asymmetry is approximately:

$$
\mathcal{A}=\frac{\left(B_{s}^{0} \rightarrow f\right)-\left(\overline{B_{s}^{0}} \rightarrow f\right)}{\left(B_{s}^{0} \rightarrow f\right)+\left(\overline{B_{s}^{0}} \rightarrow f\right)}=R \cos x_{s} t \mp \sqrt{1-R^{2}} \cos \left(2 \beta_{s}+\gamma \pm \delta\right) \sin x_{s} t
$$

here, $f$ refers to the two final states $D_{s}^{ \pm} K^{\mp}$, which correspond to the \pm terms in the $\sin x_{s} t$ term; $x_{s}$ is the $B_{s}$ mixing frequency; $2 \beta_{s}, \sim 0$ in the standard model, is the $C P$ violating phase in $B_{s}$ mixing; $\delta$ is the unknown strong phase difference; $R$ is related to the ratio of Cabibbo suppressed and allowed decays and is determined from the $\cos x_{s} t$ mixing term. Numerically, $R \sim 0.74, \sqrt{1-R^{2}} \sim 0.67$, is expected, assuming central values for the Wolfenstein parameters. If the width difference $\Delta \Gamma_{s}$ is large, then there is an additional asymmetry for the total (untagged) $B_{s}^{0}+\overline{B_{s}^{0}}$ decay rates that may allow resolution of discrete ambiguities. In toy Monte Carlo studies, depending on the input values of $\gamma, \delta$, $x_{s}, \Delta \Gamma_{s}$, and $R$, both $\mathrm{BTeV}$ and LHCb estimate similar uncertainties of $6-15^{\circ}$ on $\gamma$.

An analogous mode, $B^{0} \rightarrow D^{(*) \pm} \pi^{\mp}$ has also been modeled. Here the expected statistics are much higher, but the Cabibbo suppression is such that $R \sim 1$, and $\sqrt{1-R^{2}}$ cannot be determined from the mixing term, but must be input using Wolfenstein parameter estimates. The net $C P$ asymmetry, before flavor tagging dilution is included, is tiny, 
$\sqrt{1-R^{2}} \sim 0.016$. Nevertheless, LHCb estimates uncertainties on the order of $15-30^{\circ}$ per year on the angle $2 \beta+\gamma$.

(2) $\gamma$ from $B^{0} \rightarrow \pi^{+} \pi^{-} \pi^{0}$ : This mode measures the combination $2(\beta+\gamma)=2 \alpha$ via both $\cos 2 \alpha$ and $\sin 2 \alpha$. The decays into $\rho \pi$ modes are used to determine five complex decay amplitudes together with the $C P$ asymmetry angle $2 \alpha$. BaBar has estimated an uncertainty of order $\sigma(\alpha) \sim 5^{\circ}$ for a 2000 event sample [i3in]. From toy Monte Carlo scans, $\mathrm{BTeV}$ and $\mathrm{LHCb}$ expect errors of $10^{\circ}$ and $2.5-4.9^{\circ}$ per year, respectively. The advantage of the hadron collider experiments over the $B$ factories is high statistics, assuming that combinatoric backgrounds can be controlled.

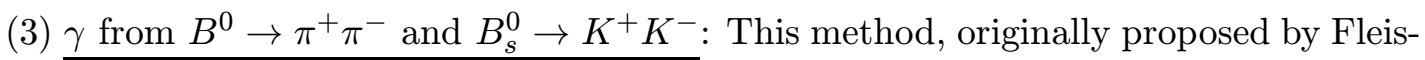
cher $[\overline{4}]$, uses the time dependent $C P$ asymmetries in these two modes, together with independently measured values of $2 \beta, 2 \beta_{s}$, and $x_{s}$ to determine $\gamma$ and one complex strong amplitude. Both tree and penguin amplitudes contribute to each of the two decays

$$
\begin{aligned}
& B^{0} \rightarrow \pi^{+} \pi^{-} \sim T+\lambda P, \\
& B_{s}^{0} \rightarrow K^{+} K^{-} \sim \lambda T+P .
\end{aligned}
$$

The crucial assumption is that the ratio $\frac{P}{T}$ is the same for both (U-spin symmetry). The experimental measurements are the time-dependent asymmetries for the two decays, both "direct" $(\cos x t)$ and "mixed" $(\sin x t)$. The very different time dependences $\left(x_{d}<<x_{s}\right)$ allow separation of the two asymmetries. At CDF the strategy is to first determine the fractions of the different two-body decay channels- $\pi^{+} \pi^{-}, K^{+} K^{-}, K^{ \pm} \pi^{\mp}$ - using relativistic rise $d E / d x$ on the untagged sample, and then use this constraint to normalize the measured asymmetries in the flavor tagged sample. The trigger is provided by the silicon vertex detector. The main issue experimentally, besides the trigger performance, is combinatoric backgrounds from ordinary QCD processes with mismeasured vertices.

$\mathrm{CDF}$ has estimated the precision on $\gamma$ assuming nominal branching fractions and QCD combinatoric backgrounds for the different modes, and nominal inputs for the external variables $\beta$ and $x_{s}$ and the internal variables $\gamma$ and $\frac{P}{T}$. The sensitivity to nominal parameter values is obtained from toy Monte Carlo experiments, and the U-spin symmetry is allowed to be broken within a physically reasonable $(20 \%)$ range. This results in an estimate of $\sigma_{\gamma} \simeq \pm 7^{\circ} \pm 3^{\circ}$ (U-spin) for $2 \mathrm{fb}^{-1}$. The LHC projections are quite similar, both in reach and in the effects of U-spin breaking. The combined error for LHCb, ATLAS, and CMS is estimated at $\sim 4.8^{\circ} /$ year for $x_{s}=30$. It should be noted that the U-spin assumptions can be constrained from measurements of the branching ratios for these modes. Since this determination involves penguin amplitudes, unlike $B_{s} \rightarrow D_{s}^{ \pm} K^{\mp}$, it is sensitive to possible new physics.

\section{4. $B_{s}$ Mixing}

Since the $B_{s}$ system is unique to hadron colliders, the physics goals are to measure in detail all features of the $B_{s}$ mixing amplitude $M_{12}-i \frac{\Gamma_{12}}{2}$,including $x_{s}=\frac{\Delta M_{s}}{\Gamma_{s}}, \Delta \Gamma_{s}$, and $\sin 2 \beta_{s}$. New physics is not expected in the $\Gamma_{12}$ amplitude, but could show up in $M_{12}$, for example anomalously large $x_{s}$ or $2 \beta_{s}$. The first critical measurement is the mixing frequence $x_{s}$; 
the ratio $\frac{\Delta M_{d}}{\Delta M_{s}}$ will constrain one side of the unitarity triangle $\left(\frac{\mid V_{t d \mid}}{\left|V_{t s}\right|}\right)$ to within about $6 \%$, limited by theoretical uncertainties.

Indirect fits to the CKM matrix predict $x_{s} \sim 26$, with $15.0<\Delta M_{s}<41.3 \mathrm{ps}^{-1}[\bar{j}]$. To measure such a rapid oscillation frequency, excellent decay time resolution is essential. For example, using semileptonic decays, $B_{s} \rightarrow D_{s} \ell \nu$, introduces large smearing of the time resolution due to the missing neutrino momentum, with the result that only very short decay-time events are useful. The method of choice is to use fully reconstructed decays such as $B_{s}^{0} \rightarrow D_{s}^{-} \pi^{+}$. To trigger on these events efficiently, CDF, BTeV, and LHCb will rely on secondary vertex triggers. D0, CMS, and ATLAS have considered lepton triggers used as flavor tags, followed by a search for the second $B$ decay to the hadronic final state. The experimental technique involves a scan of the amplitude $\mathcal{A}$ for $B_{s}^{0} \rightarrow \overline{B_{s}^{0}}=1-\mathcal{A} \cos x_{s} t / \tau$ as a function of the mixing frequency $x_{s}$. With expected time resolution of $45 \mathrm{fs}, \mathrm{CDF}$ projects an ultimate $\left(2 \mathrm{fb}^{-1}\right)$ reach of $x_{s} \sim 60$ for a 5 - $\sigma$ observation with samples of 30,000-90,000 events; this takes into account uncertainties on signal-to-background, flavor tagging efficiency, and decay branching ratios. It is worth noting that the statistical error on $x_{s}$ itself is related to the statistical error on the observed mixing amplitude; for a $5-\sigma$ significance on the amplitude, the error on $x_{s}$ is $\sigma\left(x_{s}\right)=\frac{1}{5 \sqrt{2}}$, and $\frac{\sigma\left(x_{s}\right)}{x_{s}}<1 \%$ for large $x_{s}$. That is, once a significant oscillation is observed, it is measured over many cycles and the frequency uncertainty is very small. $\mathrm{BTeV}$ and $\mathrm{LHCb}$ project similar sensitivity to $x_{s}$, depending on integrated luminosity, so we expect that if $x_{s}$ is in the standard model range, it will be measured easily.

In the standard model the complex amplitudes $M_{12}$ and $\Gamma_{12}$ are expected to be proportional in magnitude and approximately in phase. The lifetime difference in the $B_{s}$ sector is expected to be much larger than for $B_{0}$ because of the much larger mixing frequency$\Delta M_{s}>\Delta M_{d}$; eg. $\frac{\Delta \Gamma_{s}}{\Delta M_{s}} \sim 0.003-0.008$, and $\frac{\Delta \Gamma_{s}}{\Gamma_{s}} \sim 0.15$. An anomalous $C P$ violating phase in $M_{12}\left(B_{s}\right)$ would show up as a $C P$ asymmetry $\sin 2 \beta_{s}$ in the self-conjugate channels $B_{s} \rightarrow J / \psi \phi$ and $B_{s} \rightarrow J / \psi \eta^{\left({ }^{\prime}\right)}$. It would also show up as a reduction in $\Delta \Gamma_{s}$ $\left(\Delta \Gamma_{s} \propto \operatorname{Re}\left(M_{12}^{*} \Gamma_{12}\right) \propto \cos 2 \beta_{s}\right) . \Delta \Gamma_{s}$ can be obtained by comparing a predominantly $C P$ even decay mode $(J / \psi \phi)$, or a pure $C P$ even mode $\left(J / \psi \eta^{\left({ }^{\prime}\right)}\right)$, with a $C P$-mixed decay mode $\left(D_{s}^{-} \pi^{+}\right)$. It can also be extracted in principle, together with $\sin 2 \beta_{s}$ and the $C P$ even/odd fractions, from a joint fit to the $B_{s} \rightarrow J / \psi \phi$ decay angular distributions. Table $\underline{2}_{1}^{2}$ summarizes the sensitivity expected for these parameters for the collider experiments.

\begin{tabular}{|l|r|r|r|r|r|l|}
\hline Exp. & $5 \sigma x_{s}$ & $\sigma_{t}(f s)$ & $\epsilon D^{2}$ & $\sigma\left(\frac{\Delta \Gamma}{\Gamma}\right)$ & $\sigma\left(\sin 2 \beta_{s}\right)$ & $\mathrm{N}(\psi \phi / \eta)$ \\
\hline CDF & 59 & 45 & 0.09 & 0.024 & 0.15 & $6000\left(2 \mathrm{fb}^{-1}\right)$ \\
BTeV & 75 & 43 & 0.10 & 0.020 & 0.033 & $9200\left(1 \mathrm{Yr} . \times 210^{32}\right)$ \\
LHCb & 75 & 43 & 0.09 & 0.014 & 0.030 & $370 \mathrm{~K}\left(5 \mathrm{Yr} . \times 210^{32}\right)$ \\
CMS & 42 & 65 & 0.61 & 0.012 & 0.030 & $300 \mathrm{~K}\left(3 \mathrm{Yr} . \times 10^{33}\right)$ \\
ATLAS & 46 & $50 / 93$ & 0.61 & 0.018 & 0.050 & $300 \mathrm{~K}\left(3 \mathrm{Yr} . \times 10^{33}\right)$ \\
\hline
\end{tabular}

Table 2: Projected reach for $x_{s}, \sin 2 \beta_{s}$, and $\frac{\Delta \Gamma_{s}}{\Gamma_{s}}$ for the hadron collider experiments. The CMS, ATLAS, and LHCb projections for $\sin 2 \beta_{s}$ and $\frac{\Delta \Gamma_{s}}{\Gamma_{s}}$ are based on a multiparameter fit to $J / \psi \phi$ alone; BTev is based on the $J / \psi \eta^{\left({ }^{\prime}\right)}$ and $D_{s} \pi$ channels; CDF is based on the $J / \psi \phi$ and $D_{s} \pi$ channels. 


\section{Rare Decays}

At hadron colliders it is generally conceded that inclusive rare decays, eg. $b \rightarrow s \gamma, s \mu^{+} \mu^{-}$ will not be extricated from backgrounds. However, the ability to trigger on the $\mu^{+} \mu^{-}$ combination (including displaced vertex triggers) gives the hadron collider experiments a clear advantage over the $B$ factories in branching ratio measurements for exclusive modes (high rates, no flavor tagging penalty).

CDF and D0 project of order 100's of rare decays in $K^{(*)} \mu^{+} \mu^{-}$and also (using conversion photons) $K^{* 0} \gamma, \phi \gamma$. BTeV and the LHC experiments project several thousand $K^{(*)} \mu^{+} \mu^{-}$per year. BTeV and LHCb also project of order $25,000 B^{0} \rightarrow K^{* 0} \gamma$ per year. Finally, with LHC at design luminosity, the combined LHC yield for $B_{s}^{0} \rightarrow \mu^{+} \mu^{-}$is expected to be of order 27 events/year for the standard model BR. Note that CMS and ATLAS depend primarily on muon triggers and are designed for $\mathcal{L}=10^{34}$, which should provide them a special advantage for this physics.

\section{Summary}

With CDF and D0 already underway at the Tevatron, we can expect a great deal of progress on $B_{s}$ mixing and on the efficacy of vertex triggers for modes like $B^{0} \rightarrow \pi^{+} \pi^{-}$. Both the very large production cross sections and the unique sensitivity to $B_{s}$ physics make the hadron collider experiments complementary to the $B$ factories. In the LHC era, we can expect the dedicated detectors, $\mathrm{BTeV}$ and $\mathrm{LHCb}$, to apply vertex triggering, excellent particle identification, and high data acquisition rates to more difficult channels like $B_{s} \rightarrow D_{s}^{-} K^{+}$and $B^{0} \rightarrow \pi^{+} \pi^{-} \pi^{0}$. Finally, with design LHC luminosity, we can expect clear observation and study of exclusive rare decays.

\section{Acknowledgments}

I wish to thank Guy Wilkinson, Robert Kutschke, Sheldon Stone, Harold Evans, Francesco Tartarelli, Alessandra Caner, as well as my colleagues on CDF, for help in preparing this talk.

\section{References}

[1] J. Baines et al., B Decays at the LHC, CERN-TH/2000-101, hep-ph/0003238.

[2] K. Anikeev et al., B Physics at the Tevatron: Run II and Beyond, FERMILAB-Pub-01/197.

[3] Report of the BaBaR Physics Workshop, SLAC-R-504, P. F. Harrison and H. R. Quinn, Editors.

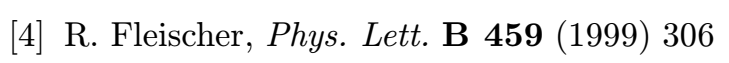

[5] A. Hocker, H. Lacker, S. LaPlace, and F. Le Dibereder, Eur.Phys.J C21 (2001) 225. 